\section{BRAZIULIAN JOURNAL \\ OF MIEDICAL AND BIOLOGICAL RESFARCH}

www.bjournal.com.br
ISSN 0100-879X

Volume 43 (03) 226-324 March 2010

BIOMEDICAL SCIENCES

AND

CLINICAL INVESTIGATION

Braz J Med Biol Res, March 2010, Volume 43(3) 310-315

Cardiac and pulmonary alterations in symptomatic and asymptomatic dogs infected naturally with Leishmania (Leishmania) chagasi

G.B.B. Alves, F.A. Pinho, S.M.M.S. Silva, M.S.P. Cruz and F.A.L. Costa

The Brazilian Journal of Medical and Biological Research is partially financed by
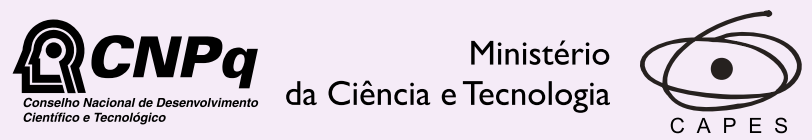

Ministério da Educação

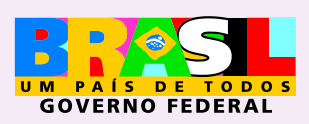

Institutional Sponsors

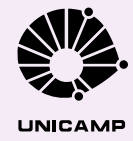




\title{
Cardiac and pulmonary alterations in symptomatic and asymptomatic dogs infected naturally with Leishmania (Leishmania) chagasi
}

\author{
G.B.B. Alves ${ }^{1}$, F.A. Pinho ${ }^{1}$, S.M.M.S. Silva ${ }^{2}$, M.S.P. Cruz ${ }^{3}$ and F.A.L. Costa ${ }^{2}$ \\ ${ }^{1}$ Programa de Pós-Graduaçao em Ciência Animal, ²Departamento de Clínica e Cirurgia Veterinária, \\ ${ }^{3}$ Departamento de Morfo-Fisiologia Veterinária, Centro de Ciências Agrárias, \\ Universidade Federal do Piauí, Teresina, PI, Brasil
}

\begin{abstract}
Fifteen symptomatic and seven asymptomatic dogs infected naturally with Leishmania chagasi were examined in order to identify the presence of parasites and changes in heart and lung. Histopathological, cytological, and immunohistochemical analyses were performed on samples of heart and lung tissues. An inflammatory reaction characterized by inflammatory mononuclear, perivascular and intermuscular infiltrates was observed in both symptomatic and asymptomatic animals on histopathological analysis of the heart. In the lung, there was thickening of the alveolar septa due to congestion, edema, inflammatory infiltrate, and fibroblast proliferation. A focal reaction was observed although a diffuse reaction was present in both groups. On cytological examination, heart and lung imprints revealed amastigotes in two symptomatic animals and heart imprints were found in 1 asymptomatic dog. Immunoperoxidase staining showed amastigotes in the lung and heart of only 1 of 6 symptomatic animals examined. Within the ethical principles and limits of this research, it can be inferred that the study of heart and lung alterations in canine visceral leishmaniasis is increasingly important for understanding the problem related to humans. Dogs with visceral leishmaniasis were a good experimental model, since infection was caused by the same agent and the animals developed clinical, pathological and immunological alterations similar to those observed in humans.
\end{abstract}

Key words: Leishmaniasis; Dog; Lungs; Heart

\section{Introduction}

In the Americas, visceral leishmaniasis is caused by the protozoon Leishmania (Leishmania) chagasi (1) and transmitted by the bite of the Lutzomyia longipalpis sandfly (2). The dog is considered to be the primary domestic reservoir of the disease $(1,3)$. However, further investigation is warranted to determine the precise role of the dog in the cycle of visceral leishmaniasis infection in endemic regions. In addition to dogs and animals of the synanthropic fauna (4), humans also seem to be reservoirs of this parasite in Brazil $(5,6)$.

In order to evaluate the role of the dog in the cycle of canine visceral leishmaniasis (CVL) infection, all manifestations of the disease must be known, ranging from clinical and pathological features to the extension and progression of lesions in various compromised organs. Dog infection with L. chagasi is clinically similar to human infection, although skin lesions are frequently found in dogs developing clinical manifestations, as well as visceral damage (7). CVL occurs as a chronic, fatal and systemic disease. The major clinical signs consist of cachexia, hypergammaglobulinemia, hepatosplenomegaly, anemia, and lymphadenopathy (7-12).

The main alterations are in organs rich in cells belonging to the mononuclear phagocyte system, such as the liver, spleen, lymph nodes, bone marrow, and gastrointestinal tract (13). However, parasite dissemination also occurs in other organs that do not belong to this system, e.g., the heart and lungs (14).

The clinical and pathological alterations due to cardiac

Correspondence: F.A.L. Costa, Setor de Patologia Animal, Departamento de Clínica e Cirurgia Veterinária, Centro de Ciências Agrárias, Campus da Socopo, Universidade Federal do Piauí, 64049-550 Teresina, PI, Brasil. Fax: +55-86-3215-5753.

E-mail: fassisle@gmail.com

Received July 25, 2009. Accepted January 4, 2010. Available online January 15, 2010. Published March 12, 2010. 
and pulmonary involvement in CVL remain largely unknown. In the heart, alterations in cardiac rhythm, necrotizing polyarteritis, granulomas, and non-suppurative myocarditis have been reported (15-18). Respiratory alterations include dyspnea, rhinitis, mucocatarrhal nasal discharge, epistaxis, stertorous breath sounds, and chronic interstitial pneumonitis $(16,19-21)$. Secondary bacterial or fungal inflammatory processes can be observed in immunosuppressive states, and pneumonia acquires a suppurative aspect (22).

The present study was conducted to assess the cardiac and pulmonary alterations in symptomatic and asymptomatic dogs naturally infected with $L$. chagasi, as well as to evaluate the nature and extension of injury to these organs, associated with the infection and the clinical form of the disease.

\section{Material and Methods}

Twenty-two privately owned or stray dogs from a Brazilian endemic area in Teresina (State of Piauí) and Timon (State of Maranhão) were included in this study. Male and female adult dogs of unknown ages and breeds were randomly tested for leishmaniasis (serology is mandatory in areas endemic for leishmaniasis) in an epidemiological survey performed by the Center for Zoonosis Control. Visceral leishmaniasis (VL) was diagnosed by positive anti-Leishmania serology combined with parasite detection. An indirect immunofluorescence assay or enzyme-linked immunosorbent assay was used for the detection of serum anti-Leishmania antibodies. For Leishmania detection, direct examination of Giemsa-stained smears of the skin, spleen and popliteal lymph nodes or culture of biopsy material collected from the sternal bone marrow, spleen, and/or popliteal lymph nodes (or all) in NNN medium (Sigma-Aldrich, USA) was performed. The animals were divided into two groups: a) 15 dogs infected naturally with L. (L.) chagasi with clinical signs of the disease and b) 7 dogs infected naturally with $L$. $(L$.) chagasi with no clinical signs of $\mathrm{VL}$.

After clinical examination, the dogs were sacrificed with an overdose of thiopental sodium (33\%, at a dose of $5 \mathrm{~mL} /$ $\mathrm{kg}, \mathrm{iv})$. All animal procedures were performed according to the Brazilian Guide for Care and Use of Laboratory Animals (Project \#3.964/97 - http://www.planalto.gov.br). All experimental protocols were previously approved by the Research Ethics Committee of Universidade Federal do Piauí, Brazil.

Following autopsy, lung and heart samples were obtained for touch imprint cytology. Samples were stained with Giemsa for parasite count in 50 random fields under the light microscope using oil immersion at a magnification of 100X. Fragments of heart and lung tissues were fixed in 10\% neutral formalin buffered with $10 \mathrm{mM}$ sodium phosphate, $\mathrm{pH}$ 7.4, for histopathological and immunohistochemical analysis. The fixed tissues were cut into $5-\mu \mathrm{m}$ sections, stained with $\mathrm{H}-\mathrm{E}$ (23) and analyzed by light microscopy for lesion classification and amastigote detection. Immunohistochemistry was performed as previously described using a mouse polyclonal anti-L. (L.) amazonensis antibody produced by the Laboratory of Seroepidemiology and Immunobiology of the Tropical Medicine Institute, University of São Paulo (24). Briefly, the antibody was obtained from BALB/c mice infected with $L$. (L.) amazonensis amastigotes and tested on formalin-fixed and paraffin-embedded sections of hamster liver infected with $L$. (L.) chagasi (positive control). Specificity was confirmed on the same sections using serum adsorbed with $L$. (L.) amazonensis promastigotes when the reactions were negative. Serum was diluted 1:1.600 (v/v) in PBS and incubated overnight at $4^{\circ} \mathrm{C}$ in a humid atmosphere. Samples were assessed using a sensitive EnVision+, peroxidase system (Dako Corporation, USA, Code K 4001) following protocols provided by the manufacturer.

Quantitative and semi-quantitative analyses were performed to measure the distribution and intensity of lesions. Lesions were classified on a scale from 0 to 5 . Data were analyzed statistically by the Mann-Whitney test. The significance level was set at $P<0.05$.

\section{Results}

Our results indicated that the most frequent clinical manifestations were local or generalized lymphadenopathy $(73.3 \%)$, skin lesions $(73.3 \%)$, fever $(46.7 \%)$, weight loss $(46.7 \%)$, apathy $(26.7 \%)$, alopecia (20\%), onychogryphosis (13.3\%), conjunctivitis (13.3\%), and anemia (6.7\%). Clinical examination did not reveal any manifestations of cardiac or pulmonary alterations. Table 1 shows the parameters investigated in the heart and lungs of each dog.

Microscopic examination of cardiac tissue revealed the presence of mononuclear perivascular and intermuscular infiltrates (Figure $1 \mathrm{~A}$ ) of minimum to moderate intensity in both asymptomatic and symptomatic animals. In one animal, a subepicardial inflammatory infiltrate predominantly composed of mononuclear cells and fatty changes was observed. In some cases, fragmentation, hyaline degeneration and necrosis of cardiac muscle fibers were observed.

Microscopic analysis of pulmonary tissue revealed thickening of the interalveolar septa due to congestion, inflammatory infiltrates and epithelial cell and fibroblast proliferation, in both asymptomatic and symptomatic animals (Figure 1E). The infiltrates were composed of mononuclear cells with a predominance of macrophages or lymphocytes and a smaller number of fibroblasts and polymorphonuclear cells. Congestion of the alveolar wall was also present. Peribronchial and peribronchiolar inflammatory infiltrates were observed, composed of mononuclear cells with invasion of the basal membrane and lamina propria. Bronchiolar exudate, hydropic degeneration and squamous metaplasia of the bronchial and bronchiolar epithelia were also observed (Figure 1G) with loss of cilia, in addition to 
thickening and hypertrophy of the peribronchial smooth muscles and thickening of the pleura. Hemosiderin was also present inside the cytoplasm of the macrophage. In one case, a thrombus was observed in a bronchial artery branch. Lesions of mild to severe intensity were mostly focal. In a few cases, lesions were diffuse. Overall, the lung had a more expressive inflammatory infiltrate than the heart (Table 1).

On cytologic examination, the presence of amastigotes in touch imprints of both the heart (Figure 1B) and lungs was an important finding (Figure 1F). Two of 15 symptomatic animals presented amastigotes in heart and lung imprints.
Amastigotes were found free in heart tissue. In the lungs, amastigotes were observed free and inside the cytoplasm of mononuclear cells of the interalveolar septa. None of the 7 asymptomatic animals showed amastigotes in lung imprints, but 1 animal exhibited leishmanias in heart tissue (Figure 1C).

The immunoperoxidase technique was performed only in 6 asymptomatic and 6 symptomatic dogs among the 22 dogs examined. Immunohistochemical analysis revealed amastigotes in the heart of only 1 of the 6 symptomatic animals examined. Only 1 was negative, and the remaining animals had minimum to moderate antigen levels (Figure

Table 1. Clinical and pathological parameters of the heart and lung of 22 seropositive dogs infected naturally with Leishmania (L.) chagasi and testing positive for the parasite.

\begin{tabular}{|c|c|c|c|c|c|c|c|c|c|}
\hline \multirow{3}{*}{ Animals } & \multicolumn{9}{|c|}{ Parameters } \\
\hline & \multirow{2}{*}{ Clinical signs } & \multirow{2}{*}{ Serology (IFI) } & \multirow{2}{*}{ Parasitology } & \multicolumn{2}{|c|}{ Cytology } & \multicolumn{2}{|c|}{$\mathrm{IMH}$} & \multicolumn{2}{|c|}{ Inflammatory infiltrate } \\
\hline & & & & Heart & Lung & Heart & Lung & Heart & Lung \\
\hline 1 & Asymptomatic & $1: 160$ & Positive & 0 & 0 & ND & ND & ++ & ++ \\
\hline 2 & Asymptomatic & $1: 320$ & Positive & 0 & 0 & + & - & ++ & ++ \\
\hline 3 & Asymptomatic & $1: 160$ & Positive & 0 & 0 & - & +++ & $\mathrm{N}$ & ++++ \\
\hline 4 & Asymptomatic & $1: 40$ & Positive & 0 & 0 & - & ++ & + & ++ \\
\hline 5 & Asymptomatic & $1: 80$ & Positive & 0 & 0 & + & ++ & + & +++ \\
\hline 6 & Asymptomatic & Negative & Positive & 0 & 0 & ++ & + & + & +++ \\
\hline 7 & Asymptomatic & $1: 80$ & Positive & 1 & 0 & +++ & ++ & + & ++++ \\
\hline 8 & Symptomatic & $1: 320$ & Positive & 1 & 3 & ++ & +++ & ++ & +++++ \\
\hline 9 & Symptomatic & $1: 80$ & Positive & 0 & 0 & + & - & ++ & ++++ \\
\hline 10 & Symptomatic & $1: 320$ & Positive & 0 & 0 & + & ++ & + & +++ \\
\hline 11 & Symptomatic & Negative & Positive & 0 & 0 & - & ++ & + & +++ \\
\hline 12 & Symptomatic & Negative & Positive & 0 & 0 & +++ & ++ & ++ & +++ \\
\hline 13 & Symptomatic & Negative & Positive & 0 & 0 & ++ & ++ & + & ++++ \\
\hline 14 & Symptomatic & $1: 80$ & Positive & 0 & 0 & ND & ND & + & +++ \\
\hline 15 & Symptomatic & $1: 160$ & Positive & 0 & 0 & ND & ND & + & ++++ \\
\hline 16 & Symptomatic & $1: 160$ & Positive & 0 & 0 & ND & ND & $\mathrm{N}$ & +++ \\
\hline 17 & vymptomatic & Negative & Positive & 0 & 0 & ND & ND & $\mathrm{N}$ & ++ \\
\hline 18 & Symptomatic & Negative & Positive & 0 & 0 & ND & ND & + & ++ \\
\hline 19 & Symptomatic & $1: 80$ & Positive & 1 & 1 & ND & ND & + & +++ \\
\hline 20 & Symptomatic & $1: 40$ & Positive & 0 & 0 & ND & ND & $\mathrm{N}$ & +++++ \\
\hline 21 & Symptomatic & Negative & Positive & 0 & 0 & ND & ND & + & ++ \\
\hline 22 & Symptomatic & ND & Positive & 0 & 0 & ND & ND & ++ & ++ \\
\hline
\end{tabular}

Serology $(\mathrm{IFI})=$ indirect immunofluorescence. Positive parasitology was determined by the presence of leishmania amastigotes in smears of bone marrow and/or lymph node. Cytology scale was the number of amastigotes in 50 random fields under a light microscope using oil immersion at a magnification of 100X. IMH = immunohistochemistry. Scale used for IMH and inflammatory infiltrate: $\mathrm{N}$ $=$ normal; $+=$ minimum or dubious; $++=$ mild $;+++=$ moderate; $++++=$ severe-moderate; $+++++=$ severe; ND $=$ not determined. 
1D). Furthermore, there was evidence of antigens in the vessel endothelium. In asymptomatic animals, 2 tested negative and 4 showed minimum to moderate antigen levels. In the lung, amastigotes were detected in only 1 animal, the same with positive cytology among 6 symptomatic animals examined. Five of these animals had mild to moderate antigen levels (Figure $1 \mathrm{H}$ ) and 1 animal tested negative. Nine animals were not tested. Among the asymptomatic animals, 5 had antigens varying from minimum to moderate in intensity and 1 animal was normal. The antigen was present in the heart myocytes and in phagocytes of the inflammatory infiltrate in the pulmonary interalveolar septa. Antigen was also present as particulate matter and found free in the interstitial space of these organs.

\section{Discussion}

It is important to be aware of the changes in body organs caused by VL, especially organs that do not belong to the mononuclear phagocyte system, in order to understand the development of the disease. Canine VL runs a chronic course in most cases and affects several vital organs, such as the heart and lungs (16). Within the ethical considerations and limits of this type of research, it can be inferred that the study of heart and lung alterations in CVL is critical for understanding the problem in relation to humans. The dog serves as an excellent model for the study of VL in humans (25) since the disease is caused by the same agent that elicits similar alterations. Injury in dogs, therefore, can be considered a parameter to be used to evaluate the disease in humans. When the disease is diagnosed early, correct treatment may be instituted.

In the present study, the animals were probably in the chronic phase of the disease. Clinical manifestations ranged from infected animals without signs of the disease to animals with full-blown disease. However, it was not possible to determine the time-course of the disease, since the animals had been infected naturally by $L$. (L.) chagasi.

In VL, cardiac involvement seems to be a rare event and the clinical manifestations due to cardiac dysfunction are not always detected. Macroscopic lesions are not always evident, requiring histopathological analysis to reveal a severe myocarditis associated with vasculitis and infarction in infected animals (17). In our investigation, despite low-grade degeneration and myocardial necrosis, the low parasite load and the discreet inflammatory response affecting a small portion of the heart tissue were probably insufficient to produce macroscopic changes and clinical manifestations that could alter myocardial function. These results differ from the intense cardiac involvement reported by López-Peña et al. (18).

In VL, tissue parasite load appears to be important in the pathogenesis of the inflammatory reaction. The parasite stimulates cellular infiltrates that determine the intensity of local injury (26). Studies have shown that amastigotes

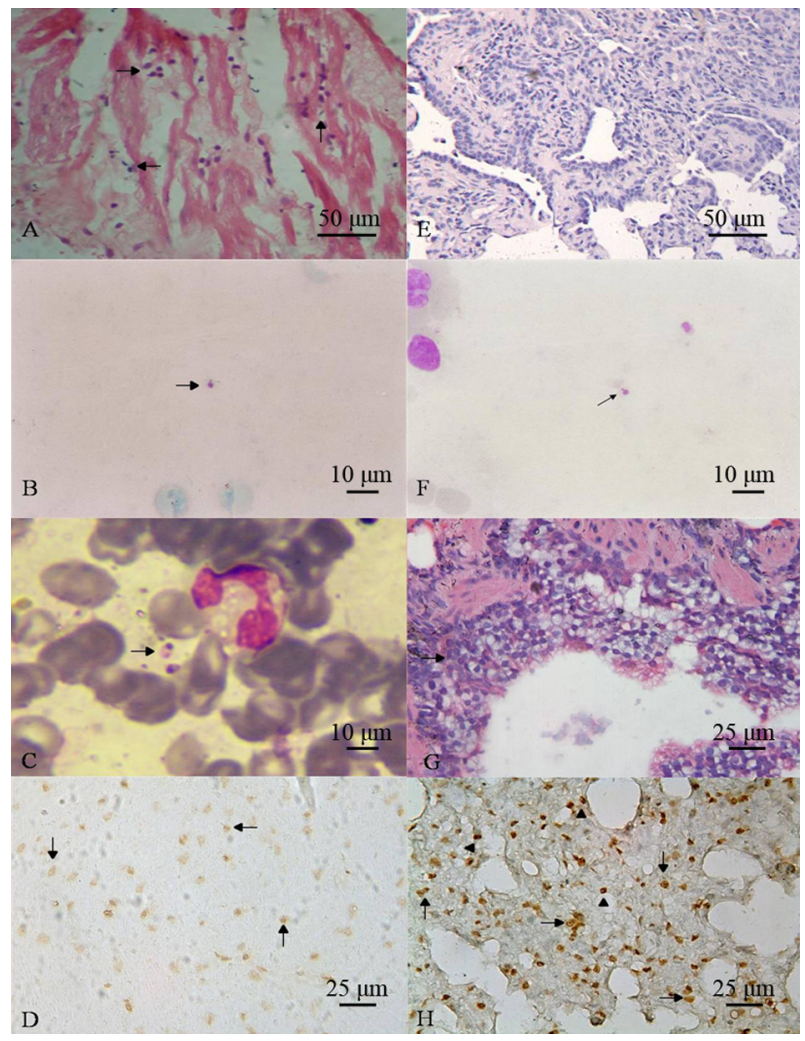

Figure 1. Histological sections from dogs infected naturally with Leishmania (Leishmania) chagasi. Heart tissue: $A$, Symptomatic dog. Mononuclear perivascular and intermuscular infiltrate (arrows). H-E staining (bar $=50 \mu \mathrm{m}) ; B$, Symptomatic dog. Amastigotes (arrow). Giemsa staining (bar $=10 \mu \mathrm{m}) ; C$, Asymptomatic dog. Amastigotes (arrow). Giemsa staining (bar $=10 \mu \mathrm{m}$ ); $D$, Symptomatic dog. Leishmania antigen (arrows). Immunoperoxidase staining (bar $=25 \mu \mathrm{m}$ ). Lung tissue (symptomatic dogs): $E$, Thickening of the inter-alveolar septa due to inflammatory infiltrate, epithelial cells and fibroblast proliferation. H-E staining $($ bar $=50 \mu \mathrm{m}) ; F$, Amastigotes (arrow). Giemsa staining (bar = $10 \mu \mathrm{m}) ; G$, Hydropic degeneration and squamous metaplasia of the bronchiolar epithelium (arrow). H-E staining (bar $=25 \mu \mathrm{m}$ ); $H$, Leishmania antigen (arrows) and amastigotes (arrowheads). Immunoperoxidase staining (bar $=25 \mu \mathrm{m})$.

may be involved in the degradation of the extracellular matrix component of the skin (27). This probably occurs in other tissues, such as the heart muscle, contributing to the development of tissue changes.

In CVL, myocarditis is characterized by an inflammatory reaction with lymphocyte, histiocyte and plasmocyte infiltrate, followed by necrosis and degeneration of cardiac muscle fibers (28). The presence of granulomas in the heart ventricle, necrotizing systemic vasculitis clinically expressed by diffuse hemorrhage in multiple organs, and thrombosis have also been observed (29-32). In the present study, however, this degree of myocardial injury was not found. Clinical and pathological manifestations of CVL seem to 
differ in endemic regions and probably depend on intrinsic factors of the vector, host, parasite, and environment, which lead to different responses concerning the extension and severity of disease.

Furthermore, lesions in organs that do not belong to the mononuclear phagocyte system do not evolve in the same manner with disease progression. However, many animals may have clinical manifestations characteristic of fully expressed disease. This fact is observed with increased parasite load and tissue damage as the disease progresses to severely compromise the immune system, predisposing the animal to secondary infections. In this study, animals that exhibited a high parasite load in organs from the mononuclear phagocyte system had a low parasite load in the heart and lung tissues.

The morphology and mechanism by which visceral leishmaniasis determines pulmonary alterations are poorly understood, particularly in cases of persistent injury. In these cases, the parasite load is very low, similar to that observed in the present study. Interstitial pneumonia or pneumonitis is the most evident lung injury in CVL, but this injury is not clinically manifested in immunocompetent dogs (22). Resistance to injuries is quite peculiar in these species. In these immunocompetent animals, pulmonary structure is characterized by a lack of secondary lobulation, a thin pleura, well-developed respiratory bronchioles, sufficiently effective collateral ventilation, and thin-walled vessels, facilitating the resolution of pulmonary lesions (33).

The major pulmonary change observed in symptomatic and asymptomatic dogs with VL is thickening of the alveolar septa due to an inflammatory response consisting of mononuclear cells, fibroblasts and congestion. Several investigators have described these well-known alterations (22,34-36).

In endemic regions for $\mathrm{VL}$, relatively frequent pulmonary lesions have been observed (Costa FAL, unpublished data), particularly in dogs originating from the city of Teresina. Autopsies were performed in the Animal Pathology Laboratory of Universidade Federal do Piauí. These alterations are different from the pattern of interstitial pneumonitis, which is easier to diagnose by microscopy. A lobar pneumonia pattern is present, as observed in animals developing full-

\section{References}

1. Lainson R, Shaw JJ. Evolution, classification and geographical distribution. In: Peters W, Lillick-kendrick R (Editors), The leishmaniasis in biology and medicine. London: Academic Press; 1987.

2. Andrade ZA, Andrade SG. [Some new aspects of the kalaazar pathology. (Morphologic study of 13 autopsy cases)]. Rev Inst Med Trop São Paulo 1966; 8: 259-266.

3. Keenan CM, Hendricks LD, Lightner L, Webster HK, Johnson AJ. Visceral leishmaniasis in the German shepherd dog. I. Infection, clinical disease, and clinical pathology. Vet blown disease. We were unable to conclude that this injury was due to a direct action of the parasite. The initial lesion may have evolved as the result of the immunosuppression caused by VL or another concomitant infection (23).

In interstitial pneumonitis, the inflammatory infiltrate seems to have an important effect on the pathogenesis of injury (37). Inflammation may be caused by leishmania and maintained by continuous antigen stimulation. Antigens reach the lungs and are detected by the immunoperoxidase technique employed in the present study. Moreover, the role of the macrophage seems to be essential in the development of lesions. Constant stimulation causes cell hyperactivity, leading to tissue injury. Therefore, exudate and cellular fibrosis appear to be the basic mechanisms responsible for thickening of focal or diffuse interalveolar septa in canine VL, as previously suggested (22).

Immunohistochemistry is a more specific and sensitive technique for parasite detection $(38,39)$ in tissue samples. However, only 1 animal in the current study showed amastigotes in heart and lung tissues. Animals examined by immunohistochemistry were fewer $(6 / 15)$ than those studied by cytology $(15 / 15)$.

Immunohistochemical analysis was fundamental to demonstrate an association between heart/lung injuries and leishmania infection. Amastigotes and leishmania antigens related to inflammatory infiltration and tissue injury were identified by this technique. In other studies, there was also evidence for the presence and involvement of the parasite as a causative agent in these lesions $(17,28,36)$.

Thus, we suggest that veterinarians become aware of potential cardiac and pulmonary involvement in the course of VL. In the present study, the duration of infection was unknown and the animals showed no signs of clinical disease. However, microscopy detected lesions in these organs that were insufficient to cause manifestation of the disease.

Cardiac and pulmonary changes related to the presence of the parasite and tissue antigens were observed here in CVL. However, these alterations did not reflect the degree of disease progression, since infection was chronic. The presence of amastigotes and antigens was observed in both symptomatic and asymptomatic dogs.

Pathol 1984; 21: 74-79.

4. Santa Rosa ICA, Oliveira ICS. Leishmaniose visceral: breve revisão sobre uma zoonose reemergente. Clin Vet 1997; 11: 24-28.

5. Costa CHN. Are dogs important reservoirs of visceral leishmaniasis in Brazil? Rev Soc Bras Med Trop 1997; 30: 155.

6. Costa $\mathrm{CH}$, Gomes RB, Silva MR, Garcez LM, Ramos PK, Santos RS, et al. Competence of the human host as a reservoir for Leishmania chagasi. J Infect Dis 2000; 182: 997-1000. 
7. Krauspenhar C, Beck C, Sperotto V, Silva AA, Bastos R, Rodrigues $\mathrm{L}$. Leishmaniose visceral em um canino de Cruz Alta, Rio Grande do Sul, Brasil. Cienc Rural 2007; 37: 907910.

8. Ciaramella P, Oliva G, Luna RD, Gradoni L, Ambrosio R, Cortese $\mathrm{L}$, et al. A retrospective clinical study of canine leishmaniasis in 150 dogs naturally infected by Leishmania infantum. Vet Rec 1997; 141: 539-543.

9. Ferrer LM. Clinical aspects of canine leishmaniasis. Proceedings of a Canine Leishmaniasis Forum. From canine leishmaniasis update. Barcelona; 1999. p 6-10.

10. Lima WG, Michalick MS, de Melo MN, Luiz TW, Luiz TW. Canine visceral leishmaniasis: a histopathological study of lymph nodes. Acta Trop 2004; 92: 43-53.

11. Langoni H, Lucheis SB, Silva RC, Castro APB, Paes AC. American visceral leishmaniasis: a case report. $J$ Venom Anim Toxins Incl Trop Dis 2005; 11: 361-372.

12. Linhares GFC, Chaves NST, Duarte SC, Fernandes PR, Amaral AVC, Souza MA. Relato de um caso clínico de leishmaniose visceral em um cão na cidade de Goiânia. Rev Patol Trop 2005; 34: 69-72.

13. Tafuri WL, de Oliveira MR, Melo MN, Tafuri WL. Canine visceral leishmaniosis: a remarkable histopathological picture of one case reported from Brazil. Vet Parasitol 2001; 96: 203-212.

14. Corbett CE, Laurenti MD. Early detection of Leishmania (Leishmania) chagasi in draining lymph node after subcutaneous innoculation in hamster. Parasitol Int 1998; 47: 307-310.

15. Blavier A, Keroack S, Denerolle PH, Goy-Thollot I, Chabanne L, Cadoré JL, et al. Atypical forms of canine leishmaniasis. Vet $J$ 2001; 162: 108-120.

16. Torrent E, Leiva M, Segales J, Franch J, Pena T, Cabrera $B$, et al. Myocarditis and generalised vasculitis associated with leishmaniosis in a dog. J Small Anim Pract 2005; 46: 549-552.

17. Zabala EE, Ramírez OJ, Bermúdez V. Leishmaniasis visceral em um canino. Ver Fac Cs Vets 2005; 46: 43-50.

18. Lopez-Pena M, Aleman N, Munoz F, Fondevila D, Suarez ML, Goicoa A, et al. Visceral leishmaniasis with cardiac involvement in a dog: a case report. Acta Vet Scand 2009; 51: 20.

19. Koutinas AF, Polizopoulou ZS, Saridomichelakis MN, Argyriadis D, Fytianou A, Plevraki KG. Clinical considerations on canine visceral leishmaniasis in Greece: a retrospective study of 158 cases (1989-1996). J Am Anim Hosp Assoc 1999; 35: 376-383.

20. Slappendel RJ. A review based on 95 cases in the Netherlands. Vet $Q$ 1998; 10: 1-16.

21. Goncalves R, Tafuri WL, Melo MN, Raso P, Tafuri WL. Chronic interstitial pneumonitis in dogs naturally infected with Leishmania (Leishmania) chagasi: a histopathological and morphometric study. Rev Inst Med Trop São Paulo 2003; 45: 153-158.

22. Slappendel RJ, Ferrer L. Leishmaniosis. In: Greene CE (Editor), Infectious diseases of the dog and cat. Philadelphia: W.B. Saunders Co. Ltda.; 1990. p 450-457.
23. Luna GL. Manual of histologic staining methods of the Armes Forces Institute of Pathology. New York: McGraw Hill; 1968.

24. Costa FA, Goto H, Saldanha LC, Silva SM, Sinhorini IL, Silva TC, et al. Histopathologic patterns of nephropathy in naturally acquired canine visceral leishmaniasis. Vet Pathol 2003; 40: 677-684.

25. Hommel M, Jaffe CL, Travi B, Milon G. Experimental models for leishmaniasis and for testing anti-leishmanial vaccines. Ann Trop Med Parasitol 1995; 89 (Suppl 1): 55-73.

26. Melo FA. Alterações da matriz extracellular na pele de cães com leishmaniose visceral naturalmente infectados. [Doctoral thesis]: Universidade Federal de Viçosa; 2005.

27. McGwire BS, Chang KP, Engman DM. Migration through the extracellular matrix by the parasitic protozoan Leishmania is enhanced by surface metalloprotease gp63. Infect Immun 2003; 71: 1008-1010.

28. Ferrari AF, Ribeiro D, Luvizotto MCR. Miocardite associada a Leishmania sp em cão - Relato de caso. Anais do $1^{\circ} \mathrm{Fo}$ rum de Leishmaniose Visceral Canina. Jaboticabal: 2006. p 48.

29. Ajello P, Catarsini O. A case of leishmaniosis in a dog with muscular and cardiac symptoms. Atti Soc Ital Sci Vet 1976; 30: 368-374.

30. Taccini E. Anatomo-histopathological findings in myocardiopathy following canine leishmaniosis. Atti Soc Ital Sci Vet 1980; 34: 271.

31. Pumarola M, Brevik L, Badiola J, Vargas A, Domingo M, Ferrer L. Canine leishmaniasis associated with systemic vasculitis in two dogs. J Comp Pathol 1991; 105: 279-286.

32. Noli C. Canine leishmaniasis. Waltham Focus 1999; 3: 1624.

33. Dungworth DL. The respiratory system. In: Jubb KVF, Kennedy PC, Palmer N (Editors), Pathology of domestic animals. New York: Academic Press; 1994. p 539-698.

34. Tryphonas L, Zawidzka Z, Bernard MA, Janzen EA. Visceral leishmaniasis in a dog: clinical, hematological and pathological observations. Can J Comp Med 1977; 41: 1-12.

35. Anderson DC, Buckner RG, Glenn BL, MacVean DW. Endemic canine leishmaniasis. Vet Pathol 1980; 17: 94-96.

36. Duarte MI, Laurenti MD, Brandao Nunes V, Rego Junior AF, Oshiro ET, Corbett CE. Interstitial pneumonitis in canine visceral leishmaniasis. Rev Inst Med Trop São Paulo 1986; 28: 431-436.

37. Quie PG. Lung defense against infection. J Pediatr 1986; 108: 813-816.

38. Giunchetti RC, Mayrink W, Genaro O, Carneiro CM, CorreaOliveira R, Martins-Filho OA, et al. Relationship between canine visceral leishmaniosis and the Leishmania (Leishmania) chagasi burden in dermal inflammatory foci. J Comp Pathol 2006; 135: 100-107.

39. Xavier SC, Chiarelli IM, Lima WG, Gonçalves R, Tafuri WL. Canine visceral leishmaniasis: a remarkable histopathological picture of one asymptomatic animal reported from Belo Horizonte, Minas Gerais, Brazil. Arq Bras Med Vet Zootev 2006; 12: 994-1000. 